\title{
Probing Nanoscale Domains of J-Aggregates Deposited on a Mica Surface
}

\author{
Serdar Özçelik, *,† Mustafa M. Demir, \\ Chemistry Department, Bilkent University, Bilkent-06533 Ankara, Turkey, and Faculty of Engineering and \\ Natural Sciences, Sabanci University Orhanll-34956,Tuzla, Istanbul, Turkey
}

Received: October 28, 2003; In Final Form: December 25, 2003

J-aggregates of 1,1',3,3'-tetraethyl-5,5',6,6'-tetrachlorobenzimidazolocarbocyanine (TTBC) were deposited on a mica surface and probed by atomic force microscopy operated at tapping mode in air. Optical spectra showed that J-aggregates were formed in aqueous solutions. Atomic force microscopy images revealed that J-aggregates deposited on mica surfaces mainly present single domains with a mean height of $2.00 \pm 0.25$ $\mathrm{nm}$ and an average diameter of $100 \pm 20 \mathrm{~nm}$. Quantitative analysis of the morphology of images indicated that the single domain of J-aggregates exhibits very uniform height and diameter distributions with polydispersity indices of 1.02 and 1.04, respectively. Based on the results, we propose a two-dimensional nanostructure in which TTBC J-aggregates could be arranged in a monolayer.

\section{Introduction}

Molecular self-assembly is a bottom-up approach to make nanoscale materials. The spontaneous self-assembly of molecules in nanoscale structures is governed by noncovalent interactions. Controlling noncovalent interactions to yield nanoscale materials with tailored properties has attracted attention due to potential technological applications. ${ }^{1}$

J-aggregate of cyanine dyes formed by short-range electrostatic interactions is an example of molecular self-assembly. The driving forces for aggregation are thought to be the dispersion forces between the nearest-neighbor molecules created by the high polarizability of the $\pi$-electrons of the polymethine backbone of cyanine dyes. ${ }^{2}$ Attachment of hydrophobic substituents to cyanine's chromophore may become significant on the arrangement of J-aggregates due to enhanced role of solvophobic forces. ${ }^{3,4}$ The degree of energy delocalization leading to exciton formation as a result of molecular arrangement determines the spectral and optical properties of J-aggregates.

The J-aggregates are the most commercially important selfassemblies as a spectral sensitizer of silver halides for the photographic industry. ${ }^{5}$ In the past decade, J-aggregates of

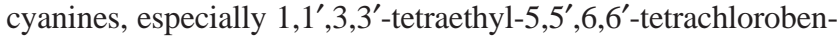
zimidazolocarbocyanine (TTBC), have been commercialized as an imaging probe in molecular cell biology. ${ }^{6}$ Furthermore, they exhibit attractive spectral and optical properties such as superradiance, superquenching, ultrafast optical switching, and electroluminescence. ${ }^{7-16}$ These properties may find applications in the field of photonic technologies.

Spectroscopic studies suggest that a few molecules are coherently coupled to form a structure that is called a coherent size anticipated to be on the scale of nanometers. On the other hand, the physical size composed of thousands to millions of molecules is generally on the micrometer scale. Many microscopy techniques had been used to probe the structure of J-aggregates. However, the quantitative analysis of morphology

* Corresponding author. Present address: Chemical Sciences Division, Pacific Northwest National Laboratory, K8-88, Richland, WA 99352. Telephone: (509) 376-4026. Fax: (509) 376-6066. E-mail: serdar.ozcelik@ pnl.gov.

Bilkent University.

$\doteqdot$ Sabancı University. is still scarce because of the widespread size and shape distribution of J-aggregates. Atomic force microscope (AFM) was employed to study the structure of J-aggregates on mica/ solution interfaces. ${ }^{17,18}$ These studies revealed that threedimensional leaflike structures were formed with the size of submicrometers and height of 3-6 $\mathrm{nm}$. Moreover, it has been shown that the aggregates were anisotropically grown on the interface. Near-field scanning optical microscopy studies demonstrated that J-aggregates in polymeric thin films form flexible molecular fibers of several micrometers long and an average width of $100 \mathrm{~nm} .{ }^{19-22}$ Supramolecular structures of J-aggregates in solutions forming ropelike superhelices of several micrometers long with a diameter of $10 \mathrm{~nm}$ were revealed by cryogenic transmission electron microscopy (cyro-TEM). ${ }^{23,24}$ On the other hand, a molecular arrangement of J-aggregates was resolved by scanning tunneling microscopy (STM). ${ }^{25}$ A linear arrangement of the molecules extended over several hundred nanometers, and $3 \mathrm{~nm}$ of interaggregate spacing was observed by STM. The linear structures were attributed to the brickwork packing arrangement originally proposed by Kuhn et al. ${ }^{26}$

Identification of the morphology of J-aggregates is of interest to predict physical as well as coherent structures and to further understand optical properties of J-aggregates which will pave the way for more scientific and technological applications. In the present study, AFM operated in tapping mode was employed to obtain structural information at nanometer scales because sample degradation is limited and the preparation is straightforward. The aim of the present study is quantitative complementation of the knowledge on the morphology of J-aggregates. The size and height of J-aggregates deposited on mica surfaces were analyzed. The AFM images revealed that J-aggregates form disk-shaped nanostructures with a mean height of $2.0 \mathrm{~nm}$ and an average diameter of $100 \mathrm{~nm}$. Polydispersity indices of the height and the diameter of J-aggregates were found to be 1.02 and 1.04, respectively. We propose that the nanostructures forming single domains are the physical size of J-aggregates containing approximately $10^{4}$ TTBC molecules.

\section{Experimental Section}

1, 1',3,3'-Tetraethyl-5, 5',6,6'-tetrachlorobenzimidazolocarbocyanine iodide salt (TTBC) was purchased from Hayashibara 

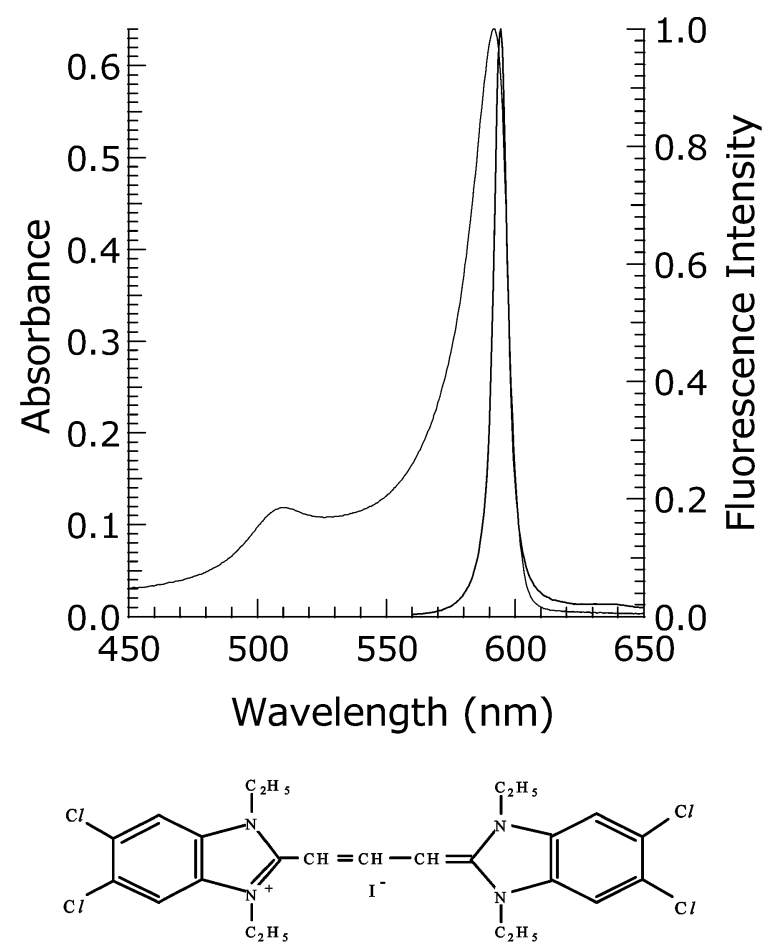

Figure 1. Absorption and fluorescence spectra of TTBC J-aggregates in $\mathrm{NaOH}$ solution. Fluorescence intensity was normalized to unity. Chemical structure of TTBC is given below the spectra.

Biochemical, Co., Okayama, Japan, and used as received. J-aggregates were prepared by mixing $1 \mathrm{~mL}$ of $1.0 \mathrm{mM}$ TTBC in methanol and $3 \mathrm{~mL}$ of $10 \mu \mathrm{M} \mathrm{NaOH}$ aqueous solution. Immediate color change of the mixture from orange to bright pink indicates the formation of aggregates. J-aggregates from aqueous solutions were deposited either by spin coating or by placing a drop of solution on mica after cleaving a fresh surface. Absorption and fluorescence spectra were recorded by Varian Cary-5 and Perkin-Elmer LS-50 spectrophotometers, respectively.

AFM images were recorded using a Nanoscope III (Digital Instruments, Santa Barbara, CA) operating at tapping mode in air. The height, phase contrast, and amplitude mode images were simultaneously recorded. An oxide sharpened Si tip with spring constant of $42 \mathrm{~N} / \mathrm{m}$ was used for all samples. In tapping mode, the cantilever oscillates at the resonance frequency of $300 \mathrm{kHz}$ in air, providing a very brief interaction of the tip with the sample surface. Images were recorded at a $256 \times 256$ pixel resolution with a scan rate of $0.6 \mathrm{~Hz}$. Typical scan area was 3 $\times 3 \mu \mathrm{m}$.

\section{Results}

Optical Spectroscopy. Formation of TTBC J-aggregates in aqueous solution was confirmed by absorption and fluorescence spectra as shown in Figure 1. The absorption spectrum of the J-aggregate showed a maximum at $592 \mathrm{~nm}$ with an asymmetric band (J-band). The asymmetric band is attributed to the presence of J-aggregates having different sizes. The asymmetric bandwidth of the J-band also manifests that the transition dipole moments could be smeared out over the lower exciton levels. A shoulder at $518 \mathrm{~nm}$ in the absorption spectrum originates from the electronic transitions in the energy levels of monomer and indicates the existence of TTBC monomers in equilibrium with the aggregates. A steady-state fluorescence spectrum showed an emission maximum at $594 \mathrm{~nm}$ with a very sharp and symmetrical band having full width at half-maximum of 8.0 $\mathrm{nm}\left(\mathrm{fwhm}=185 \mathrm{~cm}^{-1}\right)$ at room temperature. The difference in bandwidth of the absorption and emission spectra indicates that the size of excitons created by excitation and the one annihilated by emission should be different. The small Stokes shift reflects the fact that the exciton created should reach the lowest exciton level by the relaxation processes before emission.

Some estimates on the structural properties can be given using the results of the exciton theory ${ }^{27}$ for linear J-aggregates formed in solution. In the nearest-neighbor dipole-dipole interaction approximation the following equations of the exciton theory reproduce the properties of the J-band absorption spectrum:

$$
E_{k}=E_{\mathrm{o}}+2 \epsilon \cos \left(\frac{\pi k}{N+1}\right)
$$

where $N$ is the number of molecules forming the aggregate; $\epsilon$ is the interaction energy. $E_{k}$ and $E_{0}$ are, respectively, the transition energies of the $k$-exciton level and monomer.

$$
\epsilon=5.04 \mu_{\text {eff }}^{2} \frac{\left(1-3 \cos ^{2} \alpha\right)}{R^{3}}
$$

where $\mu^{2}$ eff covers constants of permittivity of vacuum and refractive index of the medium. A factor of 5.04 is needed if $\mu^{2}{ }_{\text {eff }}$ is taken in debyes squared; $R$ is in nanometers to yield $\epsilon$ in reciprocal centimeters $\left(\mathrm{cm}^{-1}\right)$.

$$
\begin{aligned}
\mu_{k}=\mu_{\mathrm{o}} \sqrt{\frac{2}{N+1}} \frac{1-(1)^{k}}{2} \cot \left(\frac{\pi k}{2(n+1)}\right) \\
k=1,2,3, \ldots, N
\end{aligned}
$$

where $\mu_{k}$ and $\mu_{0}$ are the transition dipole moments of the aggregate $k$ th excitonic transition, i.e., correspond to the $k$ th transition of eq 1 and monomer, respectively. $\epsilon$ can be expressed in terms of structural parameters: $R$ is the nearest-neighbor distance betweeen molecules in the aggregate and $\alpha$ is the angle between the molecular dipole moment and the aggregate axis.

The evaluation of eq 1 for the experimentally observed spectral position of the J-band maximum $\left(E_{k}=592 \mathrm{~nm}\right)$ together with the monomeric absorption band in methanol $\left(E_{\mathrm{o}}=515\right.$ $\mathrm{nm})^{28}$ yields $\epsilon \approx-1300 \mathrm{~cm}^{-1}$ in the large aggregate limit $(N$ $\gg 1$ ) and $\epsilon \approx-1375 \mathrm{~cm}^{-1}$ for a linear chain consisting of 10 molecules. Using eq 2 , we estimate that, for chain lengths longer than 10 molecules, the intermolecular distances of 4.5-9.5 in the corresponding angular range of $50-10^{\circ}$ can accommodate the experimental red shift in the absorption spectrum. In this estimate, we have taken $\mu^{2}=100 \mathrm{D}^{2} .{ }^{28}$ We use these numbers that translate into the coherent length of $10 \mathrm{~nm}$ as a reasonable lower limit. Details of these estimates can be found in ref 28 .

Atomic Force Microscopy. The height and lateral size measurements for individual J-aggregates were carried out by AFM and quantitatively evaluated by section analysis software provided by Digital Instruments. Figure 2 represents topography and phase contrast images recorded simultaneously and the cross sections obtained from them. The images and cross sections exhibit evidence that mica provides a very flat surface with a roughness about $0.4 \mathrm{~nm}$. The phase contrast image exhibiting excellent correlation with the topographic image is not only based on the height topography but also on other properties such as hardness and elasticity of sample, and the chemical nature of components in the sample. ${ }^{29}$ In control experiments, $10 \mu \mathrm{M}$ $\mathrm{NaOH}$ solution without TTBC was deposited on mica surface, and no particle formation was found on mica surface, just freshly cleaved. On the other hand, when the J-aggregate formed in solution, confirmed by absorption and fluorescence measure- 


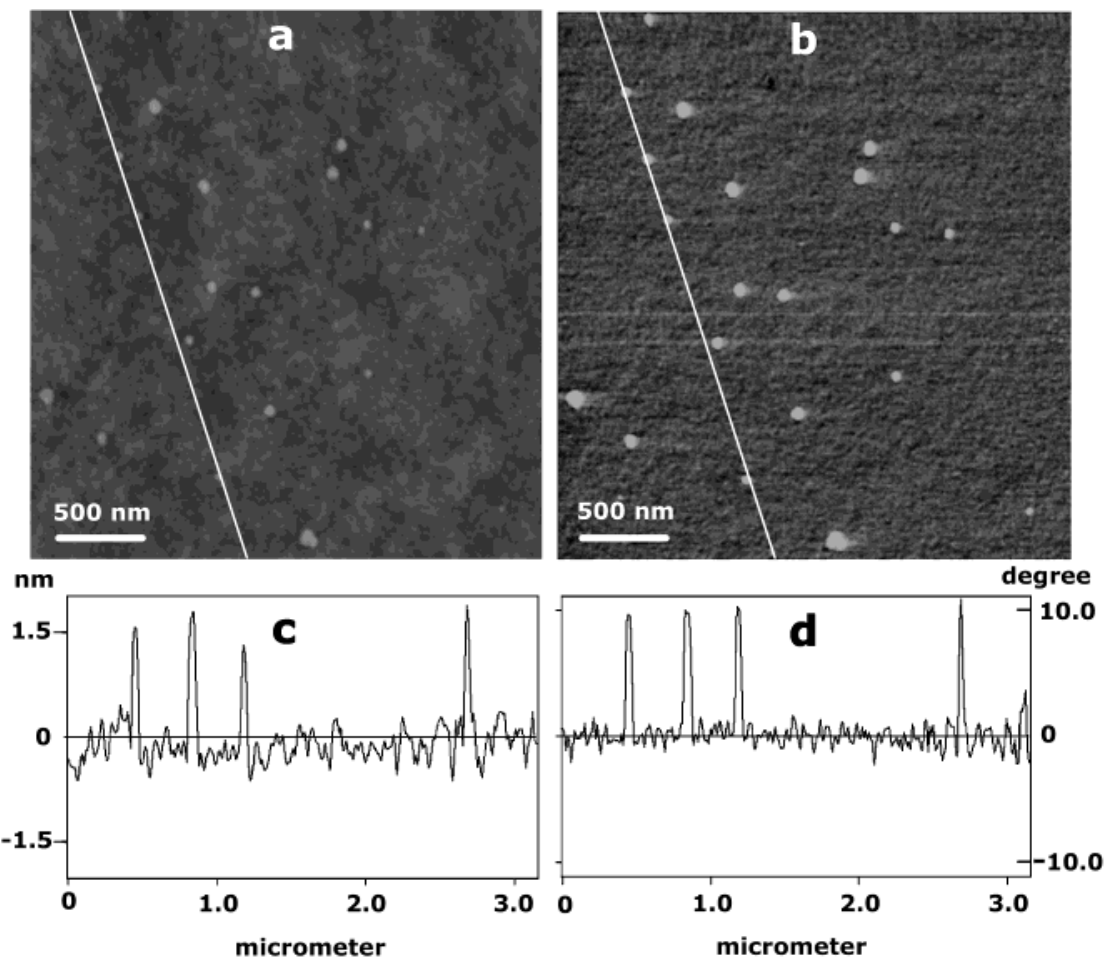

Figure 2. AFM images illustrating the topography of J-aggregates deposited on a mica surface. (a) Height image; (b) phase contrast image. The image area is $3 \times 3 \mu \mathrm{m}$. The cross-sectional profiles of the single J-aggregate domains along the lines are presented in (c) and (d).

ments, was dispersed on mica surface, disklike objects were observed. As a result, they were attributed to single domains, i.e., islands of J-aggregates deposited on mica surface, because TTBC molecules differ from the surface in topography and chemical nature. The topographic cross sections of four individual islands of J-aggregates shown in Figure 2c,d clearly demonstrate that J-aggregates form single domains with a lateral size in the range from 100 to $200 \mathrm{~nm}$ and height from 1.5 to $2.5 \mathrm{~nm}$. The actual lateral diameter (size) should be lower than the measured value due to limiting radius of the curvature of the cantilever tip, approximately $20 \mathrm{~nm}$. The lateral size is considered as the physical size of J-aggregates composed of many closely spaced "coherent sizes" in the single domain of J-aggregates. Topographic characterization and evaluation of coherent sizes in a single domain are limited because the coherent size of J-aggregates estimated from absorption spectrum is on the order of a few nanometers.

We determined heights of a large number of single domains and constructed a histogram shown in Figure 3. The data in the height histogram are fitted to a single Gaussian distribution centered at $1.96 \mathrm{~nm}$ with a variance of $0.25 \mathrm{~nm}$. It indicates that the major portion (50\%) of the J-aggregates deposited on the mica surface has an average height of $2.00 \pm 0.25 \mathrm{~nm}$. The height measurements agree very well with the crystal structure of TTBC..$^{30}$ The findings state that the J-aggregates are deposited as a single monolayer on a mica surface. On the other hand, a multilayer formation is possible only if the molecular plane of TTBC makes a minimal angle with the mica surface. Multilayer formation with a stabilizing region between the layers reducing repulsive interactions as well as steric constraints has been proposed for the mesoscopic J-aggregates in solutions. ${ }^{31,32}$

Figure 4 shows the size (the lateral diameter) histogram of the disk-shaped J-aggregates. The cross-sectional analysis provides data to a Gaussian fitted histogram yielding a mean value of $120 \mathrm{~nm}$ with a variance of $20 \mathrm{~nm}$. The lateral size can be overestimated due to the geometry and finite size effects of the tip, i.e., tip convolution. To estimate the real diameter of

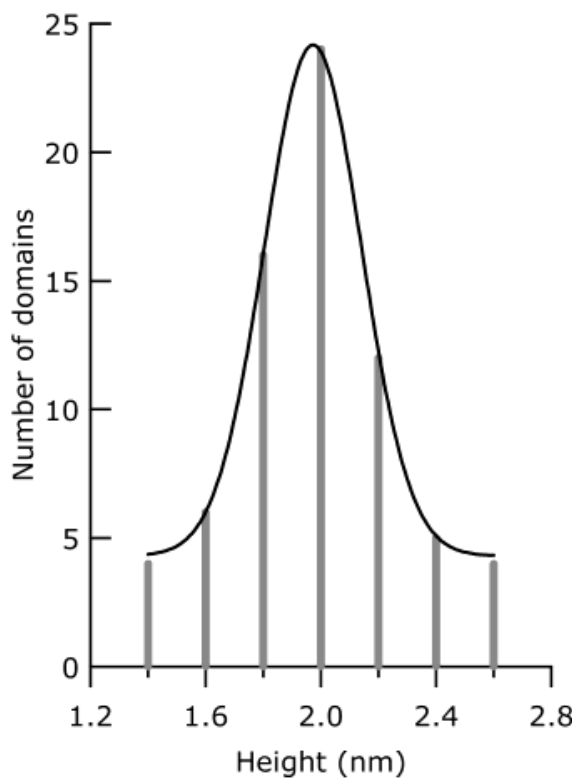

Figure 3. Height histogram of single domains of J-aggregates obtained from AFM images of more than 70 individual domains under the same scanning conditions. The solid line represents a fit of the data to a Gaussian distribution centered at $2.00 \pm 0.25 \mathrm{~nm}$. Polydispersity index of the height distribution is 1.02 .

individual J-aggregate domains, the following equation is employed. $^{33}$

$$
W_{i}=2\left(2 R Z_{i}-Z_{i}^{2}\right)^{1 / 2}+L_{i}
$$

Here, $W_{i}, Z_{i}$, and $L_{i}$ are, respectively, the apparent lateral size, height, and real diameter of the particle and $R$ is the radius of the tip. The real diameter of individual J-aggregate domains is estimated to be $100 \mathrm{~nm}$ when an apparent lateral size of 120 $\mathrm{nm}$ and a height of $2.0 \mathrm{~nm}$ are taken. The tip size of $20 \mathrm{~nm}$ was used for the calculation. It should be noted that the estimation 


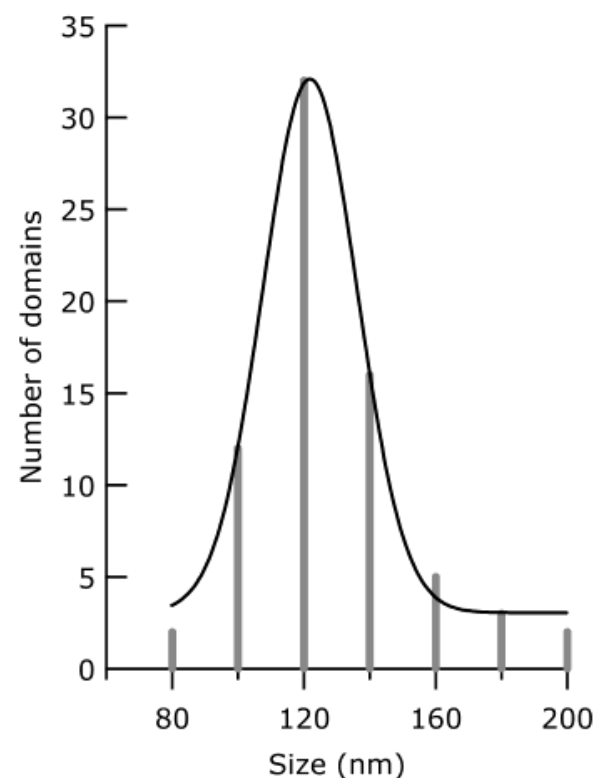

Figure 4. Lateral size (diameter of the disk-shaped single domain) histogram of J-aggregates obtained from AFM images of more than 70 individual domains under the same scanning conditions. The solid line represents a fit of the data to a Gaussian distribution centered at $100 \pm 20 \mathrm{~nm}$. Polydispersity index of the size distribution is 1.04 .

may have limitations because it considers only geometric effect but does not take into account the interactions between the tip and sample. Nevertheless, there is a slight difference between the apparent and the calculated size of the aggregates. The estimated physical size (the lateral size of the single domain) of the J-aggregates by AFM is still substantially larger than the coherent size approximated from the absorption spectrum. It is worthwhile emphasizing that the coherent size estimated may carry some uncertainty as well.

Polydispersity index (PI) is used in the context of providing a quantitative measure of the distributions. The polydispersity index is calculated using PI $=W(d) / N(d)$, where $W(d)$ and $N(d)$ are the weight average and the number average of height (size), respectively, according to eqs 5 and 6 .

$$
\begin{array}{r}
W(d)=\frac{\sum_{i} n_{i} d_{i}^{2}}{\sum_{i} n_{i} d_{i}} \\
N(d)=\frac{\sum_{i} n_{i} d_{i}}{\sum_{i} n_{i}}
\end{array}
$$

where $n_{i}$ is the number of domains of J-aggregates and $d_{i}$ is the dimension as height or lateral size of J-aggregate $i$. The same equations calculating the weight-average and number-average distributions were used for laponite nanoparticles ${ }^{34}$ and for polymers. ${ }^{35}$ The analyses resulted in polydispersity indices of 1.02 and 1.04 for the height and size, respectively. These results strongly confirm that the single domains of J-aggregates were uniformly deposited on mica surfaces.

\section{Discussion}

There is a consensus on that the molecular structure of the dye and the short-range intermolecular interactions among the molecules determine the molecular arrangement and therefore the optical properties of J-aggregates. ${ }^{2,36}$ However, the morphology of J-aggregates remains equivocal because of the difficulty of detecting unambiguous structures at nanometer scales and the nonuniformity of the structures. The single-crystal structure of TTBC provides that TTBC molecules are packed plane to plane and end to end on the long molecular edge in sheets. ${ }^{30}$ This packing is also known as the two-dimensional brickwork arrangement. ${ }^{26}$ The sheets containing the cationic part of the dye are separated by approximately $1.1 \mathrm{~nm}$ thick regions and are composed of the counterions and methanol or acetonitrile molecules. These crystallographic data also showed that TTBC occupied $2.1 \mathrm{~nm}$ linear space and covered an area of $74 \AA^{2}$. Recent studies using synchrotron X-ray diffraction and scattering suggest the formation of mesoscopic structures of J-aggregates in solutions. ${ }^{31,32}$ Supramolecular structural models exhibiting liquid crystal properties were proposed in terms of alignments of monomolecular sheets of J-aggregates separated by polar regions constituted of water, hydrophilic headgroups, and counterions with a thickness from a few tens to hundreds of nanometers.

The images revealed that J-aggregates are uniformly deposited on mica surfaces as single "disk-shaped" domains with a height of $2.0 \mathrm{~nm}$ and a diameter of $100 \mathrm{~nm}$. Utilizing the average volume of a nanodisk and the volume of a single TTBC molecule (approximated $2 \times 1 \times 1 \mathrm{~nm}$ based on the crystallographic data), we estimate nearly $10^{4}$ TTBC molecules occupying the single domain of J-aggregates. The number of molecules in the coherent size estimated from absorption spectrum is in the range $10-100$, which does not agree with AFM measurements. Therefore, the size of a single J-aggregate domain should be considered as the "physical size" of Jaggregates or the macroaggregate containing mesoaggregates (representing the coherent size) proposed by Kobayashi. ${ }^{36,37}$

A magnified AFM image of a single domain of J-aggregates and a drawing showing dimensions of a model are presented in Figure 5. Since AFM measurement provides a height of only $2.0 \mathrm{~nm}$, it is more likely that J-aggregates deposited on mica surface form a monolayer instead of multilayers requiring stabilizing regions. We suggest the observed structure of TTBC J-aggregate as a "nanodisk". Combining the findings presented here and the crystallographic data of TTBC, a structural model shown in Figure 5b is proposed: J-aggregates in single domains should be arranged in a monolayer consisting of linear chains of J-aggregates. The size and shape of the aggregates deposited on mica surface guide us to deduce two-dimensional nanostructure corresponding to the physical size of the aggregate. We assume that the nanodisks accommodate linear chains of J-aggregates formed in solution before deposition. We foresee that the deposition process does not affect the aggregate structure. Unaffectness of the deposition on the aggregation process was one of the findings of a study reporting formation of porphyrin nanorods. ${ }^{38}$ It might be necessary to conduct dynamic light scattering (DLS) measurements to determine the size of the J-aggregates in solution. However, the latter study has already pointed out the difficulty in determining the size of aggregate due to the fact that the shape of aggregate in solution should be defined precisely. ${ }^{38}$ Considering the size of the aggregate determined by AFM leads us not to pursue this matter further because the size of $100 \mathrm{~nm}$ is rather small. It was concluded that the nanorods were formed in solution, and that the substrates played no role in the aggregate formation. ${ }^{38}$ Our findings are in favor of their conclusions. Furthermore, the formation of nanorods and nanodisks being independent of the 

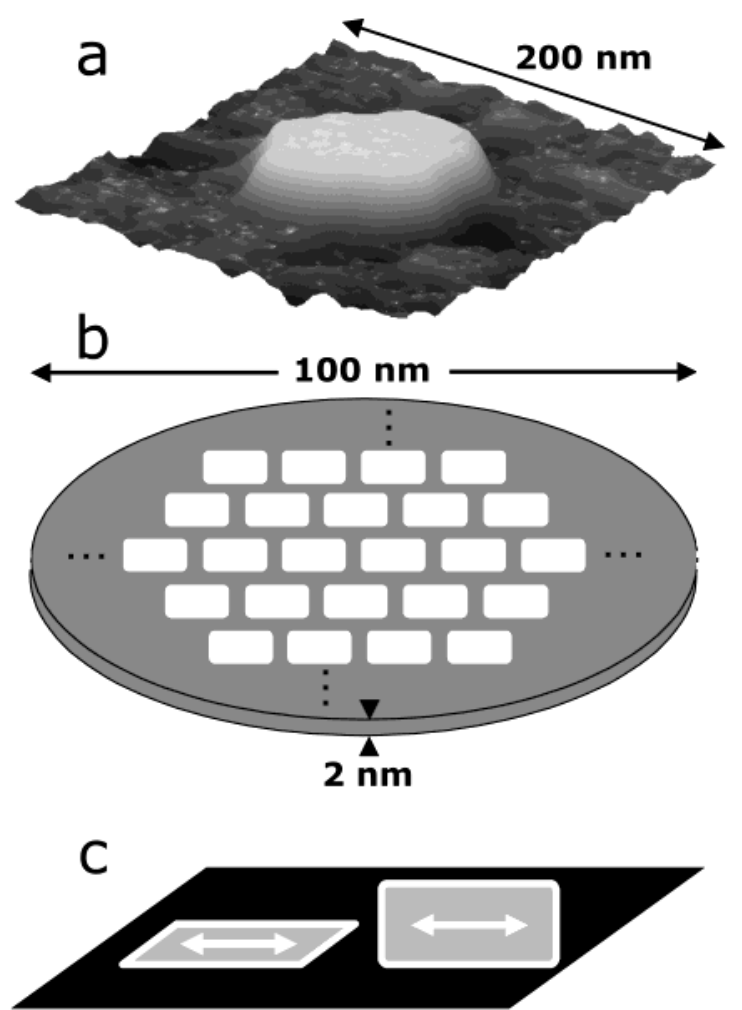

Figure 5. (a) Close-up AFM image of a J-aggregate nanodisk adsorbed on a mica surface. The image area is $200 \times 200 \mathrm{~nm}^{2}$. (b) Representation of the nanodisk with average dimensions given in nanometers and shows the proposed molecular arrangement. (c) Orientations of TTBC molecules on the mica surface.

deposition process seems to rely on the precursor structure forming aggregates. We could bear in mind the possibility of the surface-templated deposition of aggregates due to the exposed site of the negative holes because of the cleavage of mica layers. A growth scenario for aggregation on the solution/ mica interface was proposed based on AFM measurements. ${ }^{18}$

At the end, we discuss the adsorption of TTBC molecules on a mica surface. How are TTBC molecules arranged on the surface? We considered two possible arrangements of TTBC molecules on the surface (see Figure 5c): TTBC molecules could be adsorbed on (i) their molecular plane parallel to the mica surface or (ii) the molecular plane perpendicular to the surface. Since the positive charge is delocalized in the region between the two nitrogen atoms through the polymethine bridge, the interactions between the surface and TTBC molecules would be stabilized if the molecules are adsorbed on their molecular plane perpendicular to the surface. Meanwhile, the molecular plane of TTBC could form certain angles to keep the aggregate intact. TTBC molecules lying flat on the surface are less likely because the interactions between the ionic surface and the molecular plane of TTBC would be less favorable.

As a synopsis, J-aggregates on mica surfaces are mainly deposited as nanodisks with a mean height of $2.00 \pm 0.25 \mathrm{~nm}$ and an average diameter of $100 \pm 20 \mathrm{~nm}$ along with polydispersity indices of 1.02 and 1.04, respectively. Our AFM measurements and computational findings suggest that TTBC molecules in single domains could form a monolayer.

Near-field optical microscopy and spectroscopy would be very intriguing to elucidate nanoscale optical properties, especially superradiance ${ }^{7-11,39}$ and superquenching, ${ }^{15}$ of J-aggregate nanodisks deposited on various substrates. It is expected that nearfield studies would help to develop new organic optical material based on J-aggregates with enhanced and tailored optical properties. Attempts are underway to explore near-field optical properties of J-aggregates in nanodisks.

Acknowledgment. This study was supported by grants from the Scientific and Technical Research Council of Turkey (TBAG-1939) and Research Foundation of Bilkent University (CHEM-01-01). We thank Drs. Gurhan Kalay, Demet Gulen, and Vasudevan P. Biju for critical reading of the manuscript. We also thank Prof. Burak Erman for his support.

\section{References and Notes}

(1) Lehn, J.-M. Supramolecular Chemistry, Concept and Perspectives; VCH: Weinheim, Germany, 1995.

(2) Bach, G.; Dahne, S. Cyanine dyes and related compounds. In Second Supplements to the 2nd Edition of Rodd's Chemistry of Carbon Compounds; Sainsbury, M., Ed.; Elsevier: Amsterdam, 1997; Vol. IV, p 383. 7699 .

(3) von Berlepsch, H.; Kirstein, S.; Bottcher, C. Langmuir 2002, 18,

(4) von Berlepsch, H.; Kirstein, S.; Bottcher, C. J. Phys. Chem. B 2003 107,9646 .

(5) Gilman, P. B.; West, W. In Theory of the Photographic Process; James, T. H., Ed.; Macmillan: New York, 1977; p 277.

(6) (a) Smiley, S.; Reers, M.; Mottola-Hartshorn, C.; Lin, M.; Chen, A.; Smith, T. W.; Steele G. D., Jr.; Chen, L. B. Proc. Natl. Acad. Sci. U.S.A. 1991, 88, 3671. (b) Haugland, R. Handbook of Fluorescent Probes and Research Products, 9th ed.; Molecular Probes: Eugene, OR, 2002; Sections 12 and 23 and the references therein.

(7) Ozcelik, S.; Akins, D. L. J. Phys. Chem. B 1999, 103, 8926.

(8) Ozcelik, S.; Ozcelik, I.; Akins, D. L. Appl. Phys. Lett. 1998, 73, 1946

(9) Ozcelik, S.; Akins, D. L. Appl. Phys. Lett. 1997, 71, 3057.

(10) Fidder, H.; Knoester, J.; Wiersma, D. A. Chem. Phys. Lett. 1990 , 71,529

(11) Spano, F. C.; Mukamel, S. J. Chem. Phys. 1989, 91, 7988

(12) Sato, Y.; Furuki, M.; Tian, M.; Iwasa, I.; Pu, L. S.; Tatsuura, S. Appl. Phys. Lett. 2002, 80, 2254.

(13) Mal'tsev, E. I.; Lypenko, D. A.; Shapiro, B. I.; Brusentsave, M. A.; Milburn, G. H. W.; Wright, J.; Hendriksen, A.;. Berendtaev, V. I.; Kotov, B. V.; Vannikov, A. V. Appl. Phys. Lett. 1999, 75, 1896.

(14) Lidzey, D. G.; Bradley, D. D. C.; Armitage, A.; Walker, S. M.; Skolnick, S. Science 2000, 288, 1620.

(15) Jones, R. M.; Lu, L.; Helgeson, R.; Bergstedt, T. S.; McBranch, D. W.; Whitten, D. G. Proc. Natl. Acad. Sci. U.S.A. 2001, 98,14769

(16) Glaeske, H.; Malyshev, V. A.; Feller, K.-H. J. Chem. Phys. 2001 114, 1966

(17) Yao, H.; Sugiyama, S.; Kawabata, R.; Ikeda, H.; Matsuoka, O.; Yamamoto, S.; Kitamura, N. J. Phys. Chem. B 1999, 103, 4452.

(18) Ono, S.; Yao, H.; Matsuoka, O.; Kawabata, R.; Ikeda, H.; Kitamura, N.; Yamamoto, S. J. Phys. Chem. B 1999, 103, 6909.

(19) Higgins, D. A.; Barbara, P. F. J. Phys. Chem. 1995, 99, 3.

(20) Higgins, D. A.; Reid, P. J.; Barbara, P. F. J. Phys. Chem. 1996 100,1174

(21) Reid, P. J.; Higgins, D. A.; Barbara, P. F. J. Phys. Chem. 1996, 100,3892

(22) Higgins, D. A.; Kerimo, J.; vanden Bout, D. A.; Barbara, P. F. J. Am. Chem. Soc. 1996, 118, 4049.

(23) Von Berlepsch, H.; Bottcher, C.; Ouart, A,; Burger, C.; Dahne, S.; Kirstein, S. J. Phys. Chem. B 2000, 104, 5255.

(24) Von Berlepsch, H.; Bottcher, C. J. Phys. Chem. B 2000, 104, 8792.

(25) Owens, R. W.; Smith, D. A. Langmuir 2000, 16, 562.

(26) Czikkely, V.; Forsterling, H. D.; Kuhn, H. Chem Phys. Lett. 1975, 6,207

(27) Davydov, A. S. Theory of Molecular Excitons; Plenum: New York, 1970.

(28) Birkan, B. Spectroscopic and Structural Properties of J-aggregates

M.Sc. Thesis, Bilkent University, Ankara, Turkey, 2002.

(29) Maganov, S.; Reneker, D. H. Annu. Rev. Mater. Sci. 1997, 27, 175

(30) Smith, D. L.; Luss, H. R. Acta Crystallogr., Sect. B 1972, 28, 2793

(31) Harrison, W. J.; Mateer, D. L.; Tiddy, G. J. T. J. Phys. Chem. 1996, 100,2310

(32) Yao, H.; Kagoshima, Y.; Kitamura, S.; Isohashi, T.; Ozawa, Y.; Kimura, K. Langmuir 2003, 19, 8882.

(33) Eggleston, C. M. Scanning Probe Microscopy of Clay Particles; Nagy, K. L., Blum., A. E., Eds.; CMS Workshop Lectures 7; The Clay Mineral Society: Boulder, CO, 1994.

(34) Balnois, E.; Durand-Vidal, S.; Levitz, D. Langmuir 2003, 19, 6633.

(35) Rosen, S. L. Fundamental Principles of Polymeric Materials, $2 \mathrm{nd}$ ed.; Wiley-Interscience: New York, 1993; pp 53-56.

(36) Kobayashi, T. J-aggregates; World Scientific: Singapore, 1996

(37) Kobayashi, T. Supramol. Sci. 1998, 5, 343.

(38) Schwab, A. D.; Smith, D. E.; Rich, C. S.; Young, E. R.; Smith, W. F.; de Paula, J. C. J. Phys. Chem. B 2003, 107, 11339.

(39) Yaglioglu, G.; Dorsinville, R.; Ozcelik, S. J. Appl. Phys. 2003, 94, 3143. 